\title{
Dopaminergic stimulation increases selfish behavior in the absence of punishment threat
}

\author{
Andreas Pedroni • Christoph Eisenegger • \\ Matthias N. Hartmann • Urs Fischbacher • Daria Knoch
}

Received: 5 February 2013 / Accepted: 15 July 2013 /Published online: 31 July 2013

(C) Springer-Verlag Berlin Heidelberg 2013

\begin{abstract}
Rationale People often face decisions that pit self-interested behavior aimed at maximizing personal reward against normative behavior such as acting cooperatively, which benefits others. The threat of social sanctions for defying the fairness norm prevents people from behaving overly selfish. Thus, normative behavior is influenced by both seeking rewards and avoiding punishment. However, the neurochemical processes mediating the impact of these influences remain unknown. Several lines of evidence link the dopaminergic system to reward and punishment processing, respectively, but this evidence stems from studies in non-social contexts.

Objectives The present study investigates dopaminergic drug effects on individuals' reward seeking and punishment avoidance in social interaction.

Methods Two-hundred one healthy male participants were randomly assigned to receive $300 \mathrm{mg}$ of L-3,4-dihydroxypheny-
\end{abstract}

Andreas Pedroni and Christoph Eisenegger contributed equally.

A. Pedroni · M. N. Hartmann · D. Knoch

Institute of Psychology, Social and Affective Neuroscience,

University of Basel, Basel, Switzerland

C. Eisenegger $(\varangle)$

Department of Experimental Psychology, Behavioural and Clinical

Neuroscience Institute, University of Cambridge, Cambridge, UK

e-mail: christoph.eisenegger@gmail.com

U. Fischbacher

Department of Economics, University of Konstanz,

Konstanz, Germany

U. Fischbacher

Thurgau Institute of Economics, Kreuzlingen, Switzerland

A. Pedroni $(\square) \cdot$ D. Knoch $(\square)$

Social and Affective Neuroscience, Department of Psychology,

University of Basel, Birmannsgasse 8,

CH-4055 Basel, Switzerland

e-mail: andreas.pedroni@unibas.ch

e-mail: daria.knoch@unibas.ch lalanine (L-DOPA) or a placebo before playing an economic bargaining game. This game involved two conditions, one in which unfair behavior could be punished and one in which unfair behavior could not be punished.

Results In the absence of punishment threats, L-DOPA administration led to more selfish behavior, likely mediated through an increase in reward seeking. In contrast, L-DOPA administration had no significant effect on behavior when faced with punishment threats.

Conclusions The results of this study broaden the role of the dopaminergic system in reward seeking to human social interactions. We could show that even a single dose of a dopaminergic drug may bring selfish behavior to the fore, which in turn may shed new light on potential causal relationships between the dopaminergic system and norm abiding behaviors in certain clinical subpopulations.

Keywords Dopamine $\cdot$ Reward $\cdot$ Punishment $\cdot$ Normative behavior $\cdot$ Decision making

\section{Introduction}

Central to the emergence of large and complex societies has been the development of and adherence to fairness norms (Henrich et al. 2010). If these norms are violated by acting selfishly and unfairly at the cost of other people's resources, the wrongdoer is usually punished. Experimental evidence shows that a threat of punishment for defying the fairness norm sustains cooperative behavior (Fehr and Gächter 2002). Thus, the threat of punishment is a strong force in maintaining normative behavior. However, in the absence of such threat, there is a temptation to violate the fairness norm and seek personal gain. Recent experimental evidence also shows that people who are highly attracted by monetary rewards tend to violate fairness norms and follow their selfinterest (Haselhuhn and Mellers 2005). In sum, the extent to 
which an individual seeks rewards and avoids punishments is an important factor in determining normative behavior.

Despite the well-known fact that human social interaction is guided by both reward seeking and punishment avoidance (Fehr and Gächter 2002), the neurochemical underpinnings of such processes are not well understood. Dopamine is a neurotransmitter predominately associated with reward seeking (Schultz 2007), but its role in guiding behavior in the face of impending punishments is the subject of some debate (Bayer and Glimcher 2005; Salamone 1994; Ungless et al. 2004). Although dopamine neurons that respond to cues indicating punishment have long been recognized (Mirenowicz and Schultz 1996), they only recently gained more attention (Matsumoto and Hikosaka 2009; Brischoux et al. 2009; Lammel et al. 2011, 2012). The inter-relationship between reward and punishment avoidance has been accounted for by observations in animals that some of the avoidance reactions in anticipation of punishment are similar to the approach responses in anticipation of reward (Ikemoto and Panksepp 1999) and by two-factor theories of avoidance behavior (Mowrer 1947; Maia 2010) suggesting that the transition from an unsafe to a safe state is coded similarly to a reward.

In humans, psychopharmacological research generally supports a causal role of dopamine in promoting reward-seeking behavior. Administration of L-DOPA (the biochemical precursor of dopamine), augments a signal in the dopaminergically innervated reward system during reward prediction (Pessiglione et al. 2006) and increases the likelihood of choosing stimuli associated with greater reward (Bodi et al. 2009). Furthermore, a recent study showed that the administration of L-DOPA enhances the reported anticipated pleasure of positive future life events (Sharot et al. 2009). Thus, although the role of dopamine in guiding human social interaction remains elusive, these results suggest that the dopaminergic system might bias social behavior towards increasing personal monetary rewards at the cost of those of other people. For punishment avoidance, the evidence for an effect of dopaminergic pharmacology is less clear. Neuroimaging studies showed that increasing dopamine levels did not influence reward system activity for actions leading to the avoidance of punishment (Guitart-Masip et al. 2012) and did not influence the likelihood that an individual chooses stimuli associated with a smaller likelihood of punishment (Pessiglione et al. 2006). However, on a behavioral level, lowering dopamine levels using a dietary manipulation decreased the probability of a subject choosing a symbol that yielded an unexpected monetary loss (Robinson et al. 2010), which might be taken as evidence for a dopaminergic involvement in punishment avoidance.

Together, this may be taken as evidence that the dopaminergic system influences the extent to which an individual seeks rewards and avoids punishments. However, no study has addressed so far whether such influence also applies to social behavior. We thus investigated the influence of a single dose of $300 \mathrm{mg}$ of L-DOPA versus a placebo on social interaction in an economic bargaining game with two conditions, one with the presence and one with the absence of a punishment threat.

All participants who received L-DOPA or placebo were assigned the role of a proposer who has the opportunity to transfer part of an initial endowment to a recipient. Participants did this in two randomly presented conditions. In the "punishment condition", they were informed that the recipient could punish unfair transfers. Although the "punishment condition" has similarities with the ultimatum game (Güth et al. 1982), punishment in this condition is not dichotomous as in the ultimatum game but rather continuous. Thus, in this condition, the proposers face a trade-off between maximizing monetary profit and the increasing likelihood of punishment and monetary loss. Therefore, decisions are largely guided by punishment avoidance. In the other condition, the "reward condition", the recipient cannot punish unfair transfers. Here, there is a temptation to violate the fairness norm, and transfers are mostly influenced by the proposers' reward seeking. To exclude learning effects, we did not provide feedback about the recipients' punishment to the proposers until the end of the experiment. Thus, if L-DOPA administration mainly influences reward seeking during social interaction, corresponding decisions in the social interaction paradigm would be to make lower transfers in the "reward condition", compared to placebo administration. If L-DOPA increases punishment avoidance, corresponding decisions would be higher transfers in the "punishment condition".

\section{Materials and methods}

\section{Participants}

Two-hundred one healthy young male participants with a mean $( \pm \mathrm{SD})$ age of $21.9 \pm 2.3$ years took part in a doubleblind, placebo-controlled parallel session experiment which was approved by the local ethics committee. All participants provided written informed consent before participating. Four participants reported nausea, and their data were discarded, leaving a sample of 197 participants.

\section{Experimental procedure}

A total of 18 experimental sessions were conducted with an average number of 11.2 participants per session. Participants were randomly assigned to either receive a single dose of $300 \mathrm{mg}$ Madopar (consisting of $300 \mathrm{mg}$ L-DOPA and $75 \mathrm{mg}$ benserazide, a peripheral L-dihydroxyphenylalanine-decarboxylase inhibitor) $(n=101)$ or a placebo $(n=96)$. 
Upon arrival at 8:30 am, participants received a standardized meal and one serving of water and were required to ingest $10 \mathrm{mg}$ of domperidone as they indicated to have done so $12 \mathrm{~h}$ before L-DOPA administration (i.e., on the evening before the behavioral experiment). This was done to diminish possible peripheral dopaminergic side effects such as nausea and orthostatic hypotension. Immediately after, participants were asked to rate their subjective feelings on visual analogue scales (VAS, described below). After participants had read the instructions for the task, they had to answer control questions to ensure comprehension of the rules of the paradigm. Sixty minutes after L-DOPA intake, when the plasma level of L-DOPA reached its peak (Eisenegger et al. 2010), VAS were assessed again, and participants started the behavioral task (described below), which was implemented in z-Tree software and presented on computer screens (Fischbacher 2007).

Participants received a flat fee of 80 Swiss francs (1.00 $\mathrm{CHF} \approx \$ 1.00$ ) for participation in the experiment. In addition, they received a variable payoff for the bargaining game, in which 10 points were worth 0.19 CHF. Each participant received payment in private at the end of the experiment based on the points earned in the task.

\section{Social decision-making task}

Participants played four trials of two conditions of a bargaining game (Spitzer et al. 2007), where they could split an initial endowment of 100 monetary units (MUs) (equivalent to $1.90 \mathrm{CHF}$ ) between themselves (player A) and a recipient (player $\mathrm{B}$ ). The conditions were presented in random order. Participants, always playing the role of A, faced new and randomly assigned Bs in each trial.

In one condition, which we will refer to as the "punishment condition", participants were required to distribute $100 \mathrm{MUs}$ between themselves and the recipient B. They were informed that the recipient could either accept or punish unfair offers. Each player received an additional endowment of 25 MUs. B could spend all or part of the 25 MUs to reduce A's earnings. Every MU B invested into punishment led to a reduction of A's earnings by 5 MUs. For example, if A decided to keep all 100 MUs for himself and B punished maximally, then A's earnings (100 MUs initial endowment plus 25 MUs additional endowment) were reduced by 125 MUs.

In contrast to the "punishment condition," in the second condition, B was a passive recipient of A's monetary transfers. Thus, this condition lacks a punishment threat to A and therefore is similar to a dictator game. Hence, transfers are mostly influenced by the proposers' motivation to acquire monetary rewards and are only counteracted by potential fairness considerations towards the recipient. We will refer to this condition as the "reward condition."

To generate a credible punishment threat in the "punishment condition," the number of MUs that could be spent on punishment were calibrated in a previous behavioral pilot study $(n=23)$. At the end of this pilot study, the participants in the role of player B agreed that their decisions could be reused in other sessions of the experiment. In the subsequent study, each player A faced the decisions of those in the role of player B and hence faced decisions of real human opponents. Both players $\mathrm{A}$ and $\mathrm{B}$ were paid real money. However, player $B$ earned money twice, once in the pilot experiment and once in the main experiment in which he was endorsed with the average transfers of all player As.

After the bargaining game, we further elicited individuals' perception/attitudes of fairness by confronting them with a hypothetical scenario; participants were asked to indicate how many MUs they would spend to punish a proposer if he transfers 10, 20, or 50 MUs. Finally, participants filled out questionnaires, including the behavioral inhibition and behavioral activation scale (BIS/BAS) (Carver and White 1994). We assessed the BAS reward responsiveness subscale and the BIS anxiety subscale (Heym et al. 2008) to explore putative preexisting trait differences in reward and punishment sensitivity in the two treatment groups.

Measures of possible drug-related side effects

Visual analogue scales were recorded before substance administration and before the decision-making task was performed. Items in the scale were alert/drowsy, calm/excited, strong/feeble, muzzy/clear-headed, well-coordinated/clumsy, lethargic/energetic, contented/discontented, troubled/tranquil, mentally slow/ quick-witted, tense/relaxed, attentive/dreamy, incompetent/ proficient, happy/sad, antagonistic/amicable, interested/ bored, and withdrawn/gregarious. These dimensions were presented as 10$\mathrm{cm}$ lines on a computer screen, and participants marked their current states on each line with a mouse click. In line with previous studies (Chamberlain et al. 2006; Norris 1971), the factors alertness, contentedness, and calmness were calculated from these items.

\section{Data analysis}

The applicability of parametric or non-parametric statistical tests was assessed using Kolmogorov-Smirnov tests, testing for differences of normally distributed data, if not otherwise indicated. According corrections of the degrees of freedoms for violations of homogeneous/equal variances (tested with Bartlett's test and Levene's test) were applied in all parametric tests. To test transfer differences across conditions, we used a Wilcoxon signed rank-test, accounting for non-normally distributed and repeated data. To analyze drug effects on proposer transfers we applied Mann-Whitney U tests. The influence of drug treatment on participants' attitudes towards fairness was tested using a repeated measures ANOVA with hypothetical 
proposer's offers $(10,30,50$ MUS) as within-subject factors and drug treatment as between-subjects factors. This parametric test was used despite that we have non-normally distributed data in order to retain the possibility to test the interaction of drug treatment and hypothetical proposer's offers. To control for baseline measures such as drugrelated side effects and personality trait differences in BIS/ BAS, the transfers in the "reward condition" with a skewed distribution with frequent omission were transformed to frequency data (by counting the frequency of transfers greater than zero). This allowed the responses to be analyzed with generalized linear models (negative binominal distribution with a logarithmic link function), with drug treatment as a between-factor and trait measures as well as measures of drug-related side effects as covariates. Significant differences were set at $p<0.05$.

\section{Results}

We found that the punishment threat increased the proposers' transfers in the "punishment condition," compared to proposers' transfers in the "reward condition" (Wilcoxon signed rank test, $Z=11.77, p<0.001$, Fig. 1a). This shows that proposers largely disregard fairness norms in the "reward condition", but behave more norm-compliant in the "punishment condition," indicating the effectiveness of the punishment threat.

We then looked at how dopaminergic drug challenge influenced transfers in the two conditions and found that in the "reward condition," proposers who were given a placebo transferred an average of 5.00 MUs ( $\pm 11.57 \mathrm{SD})$ to the recipient. Under the influence of L-DOPA, proposers transferred only an average of 2.52 MUs $( \pm 7.69 \mathrm{SD})$ to the recipient (Mann-Whitney $\mathrm{U}$ test, $Z=2.06, p<0.039$ ). In contrast to the "reward condition," there was no significant effect of LDOPA on proposers' transfers in the "punishment condition" (Mann-Whitney U test, $Z=0.021, p<0.983$ ). Also, after controlling for transfers in the "reward condition," L-DOPA did not significantly alter the transfer differences between the "reward condition" and "punishment condition" (MannWhitney $\mathrm{U}$ test, $Z=1.245, p<0.213$ ). This additional analysis ruled out that the observed effects of L-DOPA on reward seeking (i.e., lower transfers in the "reward condition") might have counteracted the hypothesized effect of L-DOPA on punishment avoidance in the "punishment condition."

Given that distributions of transfers in the "punishment condition" and the transfers in the "reward condition" (skewed to zero transfers) were fundamentally different, it was not applicable to calculate an interaction between game condition and drug treatment, which would provide a statistical proof for the specificity of the drug-treatment effect. To furnish alternative support for the specificity of the drug treatment on behavior in the "reward condition" but not in the "punishment condition," we calculated the Bayes factor of the null hypothesis (that is, L-DOPA on proposers' transfers in the "punishment condition" has no effect) against the alternative hypothesis of drug effect, using a two-tailed Bayesian $t$ test (Rouder et al. 2009). This revealed that the data is 8.7 times more likely under the null hypothesis than under the alternative hypothesis, indicating strong evidence (Raftery 1995) for the absence of an effect of L-DOPA on transfers in the "punishment condition."

The observed dopaminergic effect was not attributable to the side effects of drug administration. There were no significant drug-treatment group differences regarding measures of
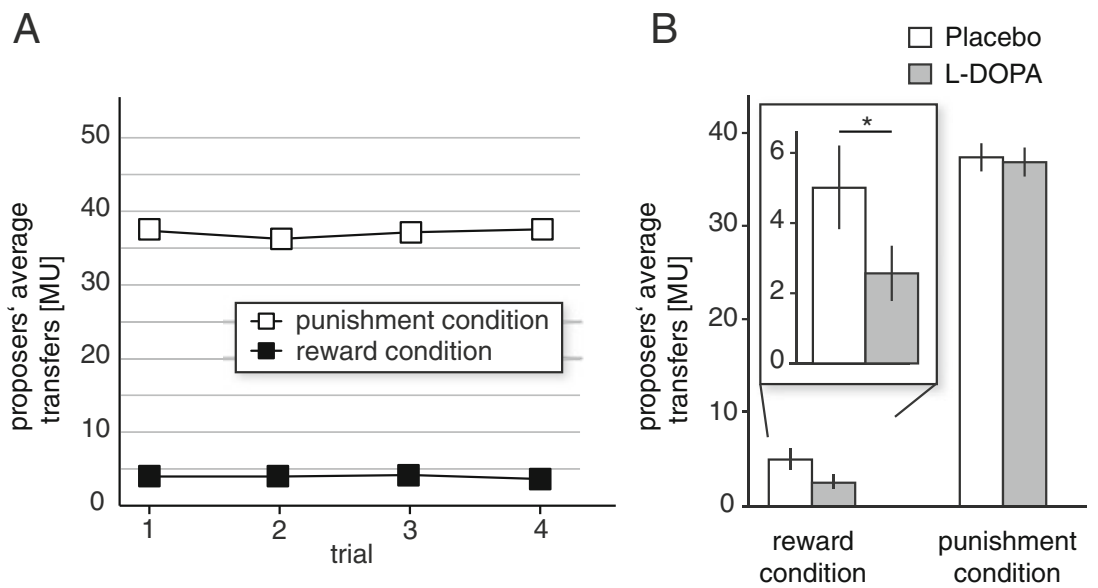

Fig. 1 Proposers' average transfers. a Proposers' average transfers to the recipient over four trials for the punishment and reward conditions. The proposers' transfer is closer to the fairness norm of equal splitting in the punishment condition, indicating the effectiveness of the punishment threat. b Proposers of the L-DOPA group made significantly smaller transfers in the reward condition compared to proposers of the placebo group. In the punishment condition, proposers' transfers did not differ significantly between the L-DOPA and placebo group, suggesting that increased availability of dopamine may enhance selfish behavior if it cannot be punished. Vertical lines indicate \pm 1 standard error of the mean 
side effects (alertness, contentedness, and calmness) between measurements at baseline and just before the behavioral task was performed (all $t \mathrm{~s}(195)<1.19$, all $p \mathrm{~s}>0.232$ ). In addition, after controlling for side effects just before the experimental task, the main effect of drug administration on transfers in the "reward condition" remained significant (generalized linear model, $\chi^{2}=6.61, p<0.010$ ).

Based on previous evidence suggesting that trait reward sensitivity is linked to decisions in similar contexts (Scheres and Sanfey 2006), we found that participants in the placebo group with high behavioral activation scale rewardresponsiveness measures (BAS RR) made fewer transfers in the "reward condition" (generalized linear model, $\chi^{2}=4.895$, $p<0.026$ ). Furthermore, BAS RR and the behavioral inhibition scale measure (BIS) did not correlate with offers in the "punishment condition", in neither experimental group (nonparametric Spearman correlations, all $\rho \mathbf{s}<0.148, p>0.150$ ).

We also tested whether the observed dopaminergic drug effect on proposer transfers could be related to pre-existing trait differences of participants in the L-DOPA and the placebo group. However, proposers in the L-DOPA group did not differ from those in the placebo group in BAS RR ( $t$ $(195)=1.532, p>0.127)$, and BIS $(t(195)=1.382, p>0.168)$. Importantly, after controlling for trait-level BAS RR and BIS in the main analysis, the effect of drug treatment on transfers in the "reward condition" remained significant $\left(\chi^{2}=4.693\right.$, $p<0.030$ ).

Finally, we tested whether an effect of L-DOPA on selfish behavior may stem from an effect on the attitude towards fairness rather than on reward seeking. Participants were asked to indicate how many MUs they would spend to punish a proposer if he transfers 10,20 , or 50 MUs. These questions were hypothetical and did not have monetary consequences. Thus, by removing monetary incentives, we remove the role of rewards during social interaction and test whether L-DOPA changed participants' attitude towards fairness. First, our results indicate that proposers were sensitive to fairness norms (repeated measures ANOVA, main effect of hypothetical offers, Greenhouse-Geisser corrected for non-sphericity, $F(1.551,302.5)=271,684$, $p>0.001)$. The amount of MUs spent to punish an unfair proposer transfer of 10 MUs was 13.0 MUs, which was significantly higher than 8.6 MUs spent to punish a proposer who transfers 30 MUs (Wilcoxon signed rank test, $Z=8.661, p<0.001$ ), which was in turn significantly higher than 1.7 MUs spent to punish fair transfers of 50 MUs (Wilcoxon signed rank test, $Z=10.389, p<0.001$ ). Furthermore, the average amount of MUs spent to punish proposer transfers in the L-DOPA group did not differ from the placebo group (repeated measures ANOVA, hypothetical offers $\times$ drug treatment, Greenhouse-Geisser corrected for non-sphericity, $F(1.551,302.5)=0.373, p>0.635)$. This suggests that the decrease in offers after L-DOPA administration in the "reward condition" is not due to induced distortions of the attitude towards fairness.

\section{Discussion}

The aim of this study was to investigate whether the administration of a dopaminergic drug influences human social interaction in the presence and absence of a threat of punishment for defying the fairness norm. Given how rewards and punishments influence normative social behavior and the involvement of dopamine in reward and punishment processing, we hypothesized that dopaminergic drug challenge would modulate social behavior in the context of rewards and punishments.

We found that a single dose of L-DOPA decreases the amount of money a proposer is willing to transfer to a recipient if he is not facing the threat of being punished for selfishness. Because the proposer can fully indulge in his self-interest to maximize monetary reward in this situation, our results support a role for the dopaminergic system in reward seeking during social interaction. Furthermore, our results suggest that the main neurochemical system involved in reward processing might facilitate selfish behavior in the absence of punishment threats.

In line with a previous study (Scheres and Sanfey 2006), the positive association of self-reported reward sensitivity with proposer transfers in the "reward condition" suggests that transfers in this condition are generally driven by the extent to which an individual seeks rewards. In keeping with our main hypothesis, we observed lower transfers following L-DOPA administration suggesting that increasing global dopamine levels which have been shown to increase reward seeking (Pessiglione et al. 2006), facilitates selfish behavior in a social context. Finally, the fact that we found no effect of L-DOPA on the answers to the hypothetical scenarios, let us rule out an alternative explanation saying that L-DOPA changed the proposers' attitude towards fairness per se.

Our data lent no support for the hypothesis that L-DOPA might increase punishment avoidance, as no significant effect on transfers in the "punishment condition" was observed. Furthermore, as we did not find a significant effect of LDOPA on the differences between transfers in the "punishment condition" and transfers in the "reward condition," we could rule out that the observed effect of L-DOPA on reward seeking (i.e., lower transfers in the "reward condition") might have obscured a putative effect of L-DOPA on punishment avoidance. By subtracting the transfers in the "reward condition" (reflecting mainly fairness considerations) from the transfers in the "punishment condition," potential fairness considerations are controlled for in the punishment condition. Thus, these differences between transfers can be interpreted as being purely driven by the proposers' attempt to maximize the 
monetary reward. This is important, as behavior which is driven by the maximization of monetary reward in the "punishment condition" should not be affected by alterations in reward seeking; on the one hand, a proposer with higher reward seeking has a higher expected utility of making a small offer because he can keep more money to himself. On the other hand, this is balanced out by a second mechanism, which is the associated loss of expected utility in case of punishment (due to the resulting reduction of monetary payoff). Thus, if reward seeking refers to the utility of the monetary income (regardless of fairness consideration) resulting from the decision about the transfer in the "punishment condition," then the extent of reward sensitivity does not affect this decision. In contrast, any alteration of punishment avoidance would affect transfers in a positive way. Hence, we conclude that L-DOPA has no significant effect on punishment avoidance during social interaction.

Our results further substantiate a role for dopamine in the generation of appetitively motivated behaviors. A recent study in a non-social context showed that L-DOPA selectively boosted striatal activity associated with actions leading to reward, but not with actions leading to the avoidance of punishment (Guitart-Masip et al. 2012). This is interesting, as it raises the question for future studies about how L-DOPA affects decision-making behavior when response requirements differ, such as when reward is attainable solely by inhibiting a response and punishment can only be avoided by an active response.

Finally, the results of this study not only emphasize a role for the dopaminergic system in human social interaction in general, but might also be relevant from a clinical perspective. For instance, Parkinson's disease patients receiving dopaminergic drugs on a regular basis might show more selfish behavior (O'Sullivan and Lees 2011) in line with the idea that (over)stimulation of reward circuits enhance the value of rewards (Dagher and Robbins 2009; Leyton et al. 2002; Rogers 2011). Furthermore, individuals with anti-social spectrum disorders show a pronounced disregard for social norms (De Brita and Hodgins 2009) and these disorders usually have a high comorbidity with drug addiction, which is in turn linked to egocentric behavior according to clinical reports (Rounsaville 2004). This further underlines a neurobiological link between the dopaminergic system and norm compliance (Buckholtz et al. 2010; Gunter et al. 2010). Interestingly, individuals affected by one of the most extreme forms of anti-social behaviors, psychopathy, seem to have elevated levels of a metabolite of the neurotransmitter dopamine in the cerebrospinal fluid (Soderstrom et al. 2001), and a recent neurochemical imaging study demonstrated that the dopaminergic reward system is hyper-responsive to monetary and drug rewards in psychopaths (Buckholtz et al. 2010). By showing that even a single dose of a dopaminergic drug may bring selfish behavior to the fore in a laboratory setting, we shed new light on potential causal relationships between the dopaminergic system and norm-abiding behaviors observed in a subset of clinical populations.

In summary, we demonstrate that L-DOPA administration decreases the amount a proposer transfers to a recipient in situations during which selfishness cannot be punished, but does not significantly affect transfers in situations with a potential punishment for selfishness. Since there is evidence from studies in a non-social context that the dopaminergic system influences the extent to which an individual seeks rewards we assume that our finding is not necessarily related to social situations alone. While our results primarily demonstrate a causal role for the dopaminergic system in reward seeking during social interaction, they also suggest that dopamine might play a relatively minor role in modulating social behavior that is guided by the avoidance of punishments. This might suggest, in turn, that punishment threats are particularly relevant to enforce fairness in individuals who have a neurobiological predisposition for reward hypersensitivity.

Acknowledgements This project was supported by a grant to DK by the Swiss National Science Foundation (PP00P1_123381). CE acknowledges support of the Swiss National Science Foundation (PA00P1_134135).

Conflict of interest The authors declare no conflict of interest.

\section{References}

Bayer HM, Glimcher PW (2005) Midbrain dopamine neurons encode a quantitative reward prediction error signal. Neuron 47:129-41

Bodi N, Keri S, Nagy H, Moustafa A, Myers CE, Daw N, Dibo G, Takats A, Bereczki D, Gluck MA (2009) Reward-learning and the noveltyseeking personality: a between- and within-subjects study of the effects of dopamine agonists on young Parkinson's patients. Brain 132:2385-95

Brischoux F, Chakraborty S, Brierley DI, Ungless MA (2009) Phasic excitation of dopamine neurons in ventral VTA by noxious stimuli. Proc Natl Acad Sci USA 106:4894-9

Buckholtz JW, Treadway MT, Cowan RL, Woodward ND, Benning SD, Li R, Ansari MS, Baldwin RM, Schwartzman AN, Shelby ES, Smith CE, Cole D, Kessler RM, Zald DH (2010) Mesolimbic dopamine reward system hypersensitivity in individuals with psychopathic traits. Nat Neurosci 13:419-421

Carver CS, White TL (1994) Behavioral inhibition, behavioral activation, and affective responses to impending reward and punishment: The BIS/BAS Scales. J Pers Soc Psychol 67:319

Chamberlain SR, Muller U, Blackwell AD, Clark L, Robbins TW, Sahakian BJ (2006) Neurochemical modulation of response inhibition and probabilistic learning in humans. Science 311:861-3

Dagher A, Robbins TW (2009) Personality, addiction, dopamine: insights from Parkinson's disease. Neuron 61:502-10

De Brita SA, Hodgins S (2009) Antisocial personality disorder. In: McMurran M, Howard R (eds) Personality, personality disorder and violence: an evidence based approach. Wiley, London

Eisenegger C, Knoch D, Ebstein RP, Gianotti LR, Sandor PS, Fehr E (2010) Dopamine receptor D4 polymorphism predicts the effect of L-DOPA on gambling behavior. Biol Psychiat 67:702-6 
Fehr E, Gächter S (2002) Altruistic punishment in humans. Nature 415:137-140

Fischbacher U (2007) z-Tree: Zurich toolbox for ready-made economic experiments. Exp Econ 10:171-178

Guitart-Masip M, Chowdhury R, Sharot T, Dayan P, Duzel E, Dolan RJ (2012) Action controls dopaminergic enhancement of reward representations. Proc Natl Acad Sci USA 109:7511-6

Gunter TD, Vaughn MG, Philibert RA (2010) Behavioral genetics in antisocial spectrum disorders and psychopathy: a review of the recent literature. Behav Sci Law 28:148-173

Güth W, Schmittberger R, Schwarze B (1982) An experimental analyses of ultimatium bargaining. J Econ Behav Organ 3:367-88

Haselhuhn MP, Mellers BA (2005) Emotions and cooperation in economic games. Brain Res Cogn Brain Res 23:24-33

Henrich J, Ensminger J, McElreath R, Barr A, Barrett C, Bolyanatz A, Cardenas JC, Gurven M, Gwako E, Henrich N, Lesorogol C, Marlowe F, Tracer D, Ziker J (2010) Markets, religion, community size, and the evolution of fairness and punishment. Science 327:1480-4

Heym N, Ferguson E, Lawrence C (2008) An evaluation of the relationship between Gray's revised RST and Eysenck's PEN: distinguishing BIS and FFFS in Carver and White's BIS/BAS scales. Pers Indiv Differ 45:709-715

Ikemoto S, Panksepp J (1999) The role of nucleus accumbens dopamine in motivated behavior: a unifying interpretation with special reference to reward-seeking. Brain Res Brain Res Rev 31:6-41

Lammel S, Ion DI, Roeper J, Malenka RC (2011) Projection-specific modulation of dopamine neuron synapses by aversive and rewarding stimuli. Neuron 70:855-62

Lammel S, Lim BK, Ran C, Huang KW, Betley MJ, Tye KM, Deisseroth $\mathrm{K}$, Malenka RC (2012) Input-specific control of reward and aversion in the ventral tegmental area. Nature 491:212-7

Leyton M, Boileau I, Benkelfat C, Diksic M, Baker G, Dagher A (2002) Amphetamine-induced increases in extracellular dopamine, drug wanting, and novelty seeking: a PET/[11C]raclopride study in healthy men. Neuropsychopharmacol 27:1027-35

Maia TV (2010) Two-factor theory, the actor-critic model, and conditioned avoidance. Learn Behav 38:50-67

Matsumoto M, Hikosaka O (2009) Two types of dopamine neuron distinctly convey positive and negative motivational signals. Nature 459:837-41

Mirenowicz J, Schultz W (1996) Preferential activation of midbrain dopamine neurons by appetitive rather than aversive stimuli. Nature $379: 449-51$
Mowrer O (1947) On the dual nature of learning - a re-interpretation of "conditioning" and "problem-solving". Harv Educ Rev 17:102-148

Norris H (1971) The action of sedatives on brain stem oculomotor systems in man. Neuropharmacol 10:181-91

O'Sullivan SS, Lees AJ (2011) Dopamine dysregulation syndrome Parkinson's disease. Blackwell, London, pp 202-214

Pessiglione M, Seymour B, Flandin G, Dolan RJ, Frith CD (2006) Dopamine-dependent prediction errors underpin reward-seeking behaviour in humans. Nature 442:1042-5

Raftery AE (1995) Bayesian model selection in social research. Sociol Methodol 25:111-164

Robinson OJ, Standing HR, DeVito EE, Cools R, Sahakian BJ (2010) Dopamine precursor depletion improves punishment prediction during reversal learning in healthy females but not males. Psychopharmacology (Berlin) 211:187-95

Rogers RD (2011) The roles of dopamine and serotonin in decision making: evidence from pharmacological experiments in humans. Neuropsychopharmacol 36:114-32

Rouder JN, Speckman PL, Sun D, Morey RD, Iverson G (2009) Bayesian $t$ tests for accepting and rejecting the null hypothesis. Psychon Bull Rev 16:225-237

Rounsaville BJ (2004) Treatment of cocaine dependence and depression. Biol Psychiat 56:803-9

Salamone JD (1994) The involvement of nucleus accumbens dopamine in appetitive and aversive motivation. Behav Brain Res 61:117-33

Scheres A, Sanfey AG (2006) Individual differences in decision making: Drive and reward responsiveness affect strategic bargaining in economic games. Behav Brain Funct 2:35

Schultz W (2007) Multiple dopamine functions at different time courses. Annu Rev Neurosci 30:259-88

Sharot T, Shiner T, Brown AC, Fan J, Dolan RJ (2009) Dopamine enhances expectation of pleasure in humans. Curr Biol 19:207780

Soderstrom H, Blennow K, Manhem A, Forsman A (2001) CSF studies in violent offenders - I. 5-HIAA as a negative and HVA as a positive predictor of psychopathy. J Neural Transm 108:869878

Spitzer M, Fischbacher U, Herrnberger B, Gron G, Fehr E (2007) The neural signature of social norm compliance. Neuron 56:185-196

Ungless MA, Magill PJ, Bolam JP (2004) Uniform inhibition of dopamine neurons in the ventral tegmental area by aversive stimuli. Science 303:2040-2 